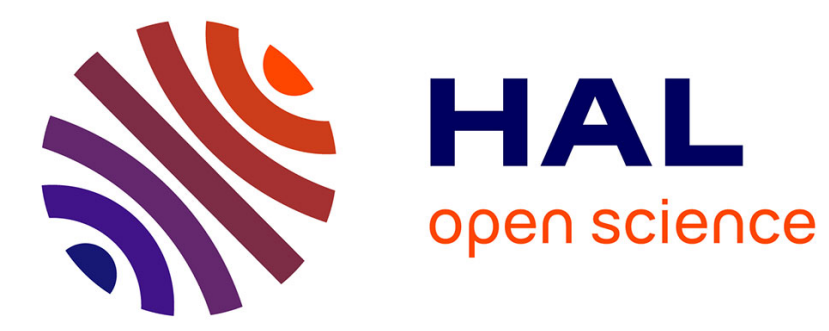

\title{
Implications in the Interpretation of Plane-Wave Expansions in Lossy Media and the Need for a Generalized Definition
}

\author{
Andréa Cozza, Lyazid Aberbour, Benoît Derat
}

\section{- To cite this version:}

Andréa Cozza, Lyazid Aberbour, Benoît Derat. Implications in the Interpretation of Plane-Wave Expansions in Lossy Media and the Need for a Generalized Definition. Wave Motion, 2017, 10.1016/j.wavemoti.2017.11.003 . hal-01589787

\section{HAL Id: hal-01589787}

https://hal-centralesupelec.archives-ouvertes.fr/hal-01589787

Submitted on 19 Sep 2017

HAL is a multi-disciplinary open access archive for the deposit and dissemination of scientific research documents, whether they are published or not. The documents may come from teaching and research institutions in France or abroad, or from public or private research centers.
L'archive ouverte pluridisciplinaire HAL, est destinée au dépôt et à la diffusion de documents scientifiques de niveau recherche, publiés ou non, émanant des établissements d'enseignement et de recherche français ou étrangers, des laboratoires publics ou privés. 


\title{
Implications in the Interpretation of Plane-Wave Expansions in Lossy Media and the Need for a Generalized Definition
}

\author{
A. Cozza ${ }^{\mathrm{a}}$, L. Aberbour ${ }^{\mathrm{b}}$, B. Derat ${ }^{\mathrm{b}}$ \\ ${ }^{a}$ Physique et Ingénierie de l'Électromagnétisme \\ Group of Electrical Engineering of Paris (GeePs), CNRS UMR 8507, CentraleSupelec - Univ Paris-Sud - UPMC \\ 11 rue Joliot-Curie, Plateau de Moulon, 91192 Gif-sur-Yvette, France. \\ Contact : andrea.cozza@centralesupelec.fr \\ ${ }^{b}$ Art-Fi S.A., 27 rue Jean Rostand, 91400 Orsay, France.
}

\begin{abstract}
Plane-wave expansions (PWEs) based on Fourier transform and their physical interpretation are discussed for the case of homogeneous and isotropic lossy media. Albeit being mathematically correct, standard Fourier-based definition leads to nonphysical properties, such as the absence of homogeneous plane waves, lack of dissipation along transversal directions and inaccurate identification of single plane waves. Generalizing the PWE definition using Laplace transform, which amounts to switching to complex spectral variables, is shown to solve these issues, reinstating physical consistency. This approach no longer leads to a unique PWE for a field distribution, as it allows an infinite number of equivalent definitions, implying that the interpretation of the individual components of a PWE as physical plane waves does not appear as justified. The multiplicity of the generalized definitions is illustrated by applying it to the near-field radiation of an elementary electric dipole, for different choices of Laplace cuts, showing the main differences in the generalized PWEs.
\end{abstract}

Keywords: Plane-wave expansion, lossy media, near-field scans.

\section{Introduction}

Expanding field distributions onto a Fourier basis is a wellestablished procedure used for solving problems of radiation and propagation [1-8]. Also known as spectral representation, it provides an interesting and effective framework, as it allows algebraic representations of integro-differential equations, which lend themselves to physical interpretation, since each basis function corresponds to the mathematical description of a plane wave. Field distributions are therefore represented as a linear combination of plane waves, where each one can be propagated through a homogeneous or layered space according to physical laws, whence their being referred to as plane-wave expansions (PWEs).

It is therefore reasonable that the individual components of a PWE are often considered as physical plane waves, regarding their propagation vectors and amplitudes as physical parameters that accurately describe the way they propagate through a homogeneous medium. While this has a physical foundation in the case of propagative contributions, as recalled in Sec. 2, the interpretation of PWE has been at the center of controversies. In $[9,10]$, the need to consider portions of a PWE not as necessarily physically consistent at the individual scale was already pointed out for reactive contributions to a PWE, when taken individually. A related issue was reported in [5, 11, 12], when considering homogeneous contributions in asymptotic expressions.

The problems at interpreting a PWE worsen when lossy media are considered. The introduction of losses is presented in the literature as not requiring any modification to the standard Fourier-based definition. Sec. 2.2 comments on some inconsistencies that make an intuitive interpretation of a PWE look dubious, e.g., the absence of any homogeneous contribution in case of lossy media. In particular, the PWE can be shown not to provide accurate identification of single plane waves, as opposed to lossless settings.

Sec. 3 studies an alternative definition of PWE based on Laplace transform. Allowing complex spectral variables, Laplace transform makes it possible again to identify single plane waves from field distributions even in lossy media. The existence of an extended region of convergence for Laplace transform implies that a field distribution does not correspond to a unique PWE, thus leading to an extended family of propagators. All of these propagators yield identical results when propagating field distributions from one plane to another. But each propagator being different it is no longer possible to associate a single common physical meaning to each individual component (or plane wave) across all PWEs.

Numerical examples in Sec. 4 illustrate the fundamental differences in the generalized PWE depending on the chosen Laplace cut, thus supporting the conclusion that the PWE cannot be interpreted, in lossy media, as composed of physical entities, but should rather be regarded as a mathematical representation. More specifically, there is a case for choosing on purpose alternative definitions when identifying single plane waves that are expected to be homogeneous on physical grounds, such as for asymptotic representations needed for far-field radiation. 


\section{Plane-wave expansion and field propagation}

In the following an $\exp (\mathrm{j} \omega t)$ time dependence will be assumed, and dropped throughout the paper for simplicity, thus working with phasor notation. The background medium is assumed to be homogeneous and isotropic, with a relative permittivity constant $\epsilon_{r}$ and an electric conductivity $\sigma$ supposed for the time being to be equal to zero, thus with a propagation constant $k_{o}$ defined as

$$
k_{o}^{2}=\omega^{2} \mu_{o} \epsilon_{o} \epsilon_{r} .
$$

In this section and the next one scalar field distributions are considered for the sake of simplicity, but the ideas discussed directly apply to vector fields as well, as done in Sec. 4.

\subsection{Standard Fourier-based definition}

The standard PWE definition is usually introduced by invoking the property of completeness of the Fourier basis $[3,4,7,8]$. A generic scalar field $u\left(\boldsymbol{R}, z_{o}\right)$ sampled at a plane $z=z_{o}$ (the scan plane), outside the source region, is projected onto $2 \mathrm{D}$ functions of the kind $f(\boldsymbol{R} ; \boldsymbol{K})=\exp (-\mathrm{j} \boldsymbol{K} \cdot \boldsymbol{R})$, with $\boldsymbol{R}=x \hat{\boldsymbol{x}}+y \hat{\boldsymbol{y}}$ the transversal position over the plane and $\boldsymbol{K}=k_{x} \hat{\boldsymbol{x}}+k_{y} \hat{\boldsymbol{y}}$ the spectral variable. Since the projection between two of the above basis functions, e.g., for two choices of $\boldsymbol{K}$, here $\boldsymbol{K}_{1}$ and $\boldsymbol{K}_{2}$, gives

$$
\iint_{-\infty}^{\infty} \mathrm{d} \boldsymbol{R} f^{*}\left(\boldsymbol{R} ; \boldsymbol{K}_{1}\right) f\left(\boldsymbol{R} ; \boldsymbol{K}_{2}\right)=\delta\left(\boldsymbol{K}_{1}-\boldsymbol{K}_{2}\right)
$$

with $*$ the complex conjugate and $\delta(\cdot)$ Dirac's delta distribution, it is indeed possible to identify precisely the coefficient associated to each basis function. The identification is exact only in the case of data gathered over an infinitely large plane, i.e., the domain over which the orthogonality relationship (2) holds.

The projection, computed using the inner product, leads to defining the complex amplitude of the Fourier transform of $u\left(\boldsymbol{R}, z_{o}\right)$ as

$$
\tilde{u}\left(\boldsymbol{K}, z_{o}\right)=\int_{-\infty}^{\infty} \int_{-\infty}^{\infty} \mathrm{d} \boldsymbol{R} u\left(\boldsymbol{R}, z_{o}\right) f^{*}(\boldsymbol{R} ; \boldsymbol{K})=\iint_{-\infty}^{\infty} \mathrm{d} \boldsymbol{R} u\left(\boldsymbol{R}, z_{o}\right) \mathrm{e}^{\mathrm{j} \boldsymbol{K} \cdot \boldsymbol{R}},
$$

and therefore to express $u\left(\boldsymbol{R}, z_{0}\right)$ as a (infinite) linear combination

$$
u\left(\boldsymbol{R}, z_{o}\right)=\frac{1}{4 \pi^{2}} \int_{-\infty}^{\infty} \int^{\infty} \mathrm{d} \boldsymbol{K} \tilde{u}\left(\boldsymbol{K}, z_{o}\right) \mathrm{e}^{-\mathrm{j} \boldsymbol{K} \cdot \boldsymbol{R}} .
$$

The above representation is known as PWE (or spectrum) or angular spectrum when expressed as a function of the director cosines of $\boldsymbol{K}$. Insofar it is essentially a mathematical procedure, with no physical-motivated rationale. The connection to plane wave propagation will be recalled in a moment.

Plane-wave expansions can be defined and computed for any plane $z$, but it can be demonstrated that PWEs for different values of $z$ are actually related to one another as

$$
\tilde{u}(\boldsymbol{K}, z)=P\left(\boldsymbol{K}, z-z_{o}\right) \tilde{u}\left(\boldsymbol{K}, z_{o}\right),
$$

where $P\left(\boldsymbol{K}, z-z_{o}\right)$ is the spectral propagator, given by

$$
P\left(\boldsymbol{K}, z-z_{o}\right)=\mathrm{e}^{-j k_{z}\left(z-z_{o}\right)} .
$$

Defining $\boldsymbol{k}=\boldsymbol{K}+k_{z} \hat{z}$, Helmholtz equation enforces the condition

$$
\boldsymbol{k} \cdot \boldsymbol{k}=k_{o}^{2},
$$

so that for each value of $\boldsymbol{K}$, i.e., for each doublet $\left(k_{x}, k_{y}\right)$, there exists a value $k_{z}=\gamma$, such that

$$
\gamma^{2}=k_{o}^{2}-\boldsymbol{K} \cdot \boldsymbol{K}
$$

The complex amplitude of each basis function $f(\boldsymbol{R} ; \boldsymbol{K})$ propagate from a plane $z_{o}$ to a plane $z$ as dictated by (6), i.e.,

$$
f(\boldsymbol{R} ; \boldsymbol{K}) P\left(\boldsymbol{K}, z-z_{o}\right)=\mathrm{e}^{-\mathrm{j} \boldsymbol{k} \cdot\left(\boldsymbol{r}-z_{o} \hat{z}\right)},
$$

with $\boldsymbol{r}=\boldsymbol{R}+z \hat{\boldsymbol{z}}$. Therefore, each of these functions behaves as a plane wave propagating according to a propagation vector $\boldsymbol{k}$. This observation is the main rationale behind interpreting a PWE as a collection of actual plane waves propagating through an homogeneous infinite medium. In (8) only the forward propagating solution is usually retained, assuming a source to be found below the scan plane $z_{o}$; this solution is characterized by the physical choice of decaying waves for an increasing $z$, i.e., with a negative imaginary part of $\gamma$.

The lossless case allows a clear classification of the $\boldsymbol{K}$-space into active (visible) and reactive (non-visible) regions, as a function of the sign of the argument of the square root in (8). In case of a positive argument, $\gamma \in \mathbb{R}$, corresponding to propagative fields structures, whereas for negative arguments, i.e., for those $\|\boldsymbol{K}\|>k_{o}, \gamma \in \mathbb{I}$, thus exponentially decaying structures.

The corollary of condition (9) is that the quantities $k_{x}, k_{y}$ and $k_{z}$ must be the Cartesian components of the vector $\boldsymbol{k}$, i.e., for each component $k_{i}$ of the vector $\boldsymbol{k}$

$$
k_{i}=\boldsymbol{k}_{o} \cdot \hat{\boldsymbol{u}}_{i},
$$

with $\hat{\boldsymbol{u}}_{i}$ the unit vector of the $i$-th Cartesian axis, as long as $\boldsymbol{K}$ belongs to the active region where they are expected to be homogeneous plane waves. If plane waves in the active region can be interpreted as representing physical rather than just mathematical objects, then they can be expected to correspond to homogeneous plane waves, as inhomogeneous ones can be observed only in case of two half spaces, of which one with negligible losses and the other one with much larger dissipation [13].

Condition (10) is indeed satisfied in the case of lossless media, since the far-field distribution generated by a source can be expressed in terms of their PWE as [5, 7]

$$
u(\boldsymbol{r})=\mathrm{j} k_{o} \tilde{u}\left(\boldsymbol{K}_{F F}\right) \frac{\mathrm{e}^{-\mathrm{j} k_{o} r}}{r} \cos \theta
$$

with

$$
\boldsymbol{K}_{F F}=k_{o} \mathbf{1}_{t} \cdot \hat{\boldsymbol{r}}
$$

and $\mathbf{1}_{t}=\hat{\boldsymbol{x}} \hat{\boldsymbol{x}}+\hat{\boldsymbol{y}} \hat{\boldsymbol{y}}$ a dyad operating a projection over the $x y$ plane; notice how (12) is equivalent to (9). These results imply that an observer is expected to experience a homogeneous locally plane wave propagating along the direction of observation, consistently with the condition of a isotropic and homogeneous medium. Hence, plane waves in the active region of the PWE can be interpreted as physical quantities in their own right. 


\subsection{Lossy media and related issues}

In case of a dissipative background medium, it is characterized by a complex relative dielectric permittivity $\epsilon_{c}=\epsilon_{r}-$ $\mathrm{j} \sigma /\left(\omega \epsilon_{o}\right)$, taking the place of $\epsilon_{r}$ in (1). In this case the dispersion relation $k=k(\omega)$ is no longer linear in $\omega$ and therefore dissipative media may be referred to as dispersive [8].

To the best of our knowledge, in case of $\sigma \neq 0$ the standard definition of PWE and propagator recalled in Sec. 2.1 are maintained throughout the literature[7, 8], by still choosing $\boldsymbol{K} \in \mathbb{R}^{2}$. It is this choice that is argued as arbitrary in the rest of this paper, and discussed as the reason for apparent, but ultimately fictitious, physical inconsistencies.

The only difference in the equations with respect to the previous case of lossless media is acknowledging that $k_{o}$ is now complex and therefore that the domain of $k_{z}$, as given in (8), can no longer be divided into purely real and imaginary regions, or active and reactive, respectively.

In fact, from a physical point of view there are two issues at stake when dealing with lossy media. First, if each basis function is to be regarded as physically meaningful, it must be consistent with the fact that each plane wave is evolving in a homogeneous and isotropic medium. Second, there exist other alternative definitions with interesting properties, as discussed in Sec. 3.

Concerning the first point, several observations suggest that the standard PWE definition leads to spectra that should not be interpreted as composed of physically meaningful plane waves, when taken individually. Eq. (11) proved that for lossless media plane waves in a PWE can be regarded as physical quantities. But it is striking that in case of lossy media, when directly applying the standard definition of PWS, the entire PWE is made up of what would be interpreted as inhomogeneous plane waves, apart for the direction of propagation normal to the $x y$ plane. Still, in a lossy medium the far-field condition would require, though asymptotically, the observation of homogeneous locally-plane waves along any direction, as long as the medium is isotropic and homogeneous. There, it would be expected that each Cartesian component $k_{i}$ of the vector $\boldsymbol{k}$ comply with (10). Since for the standard definition $\boldsymbol{K} \in \mathbb{R}^{2}$, the projection of a complex $k_{o}$ over the $x y$ plane should also be complex. In other words, the standard choice of $\boldsymbol{K} \in \mathbb{R}^{2}$ requires that each plane wave propagates transversally over the $x y$ plane without losses, while dissipation occurs only for longitudinal propagation along $z$.

It should be stressed that although not physically consistent with homogeneous media, inhomogeneous plane waves are allowed by (7), as they represent exact solutions of Helmholtz equation.

It appears that only [7] has studied how (11) must be modified in order to take into account the case of lossy media, and restore the necessary homogeneity of the local plane waves observed in the far-field region of a source. What was proven in [7, Sec. 3.3] is that (11) and (12) are still valid in the case of lossy media, even though now $k_{o} \in \mathbb{C}$, i.e., the far-field radiation is related to the PWE sampled not over $\boldsymbol{K} \in \mathbb{R}^{2}$, but rather over complex values. This result seems in contradiction with the standard Fourier-based definition of the PWE. It can be remarked how (12) automatically enforces (10), resulting in the use of purely homogeneous plane-wave contributions from the PWE, as opposed to the standard PWE definition limited to $\boldsymbol{K} \in \mathbb{R}^{2}$. The implications of sampling a PWE for a complex $\boldsymbol{K}$ will be discussed in Sec. 3 .

Another limitation of the standard PWE definition is its inability to correctly assess the parameters of a plane wave propagating through a lossy medium. Suppose a single plane wave propagates in a medium with $k_{o} \in \mathbb{C}$, along a direction $\hat{\boldsymbol{k}}_{p}$, with a complex amplitude $A_{p}$. In the case of a lossless medium, the PWE would be capable of immediately identify the plane-wave parameters, since for $k_{o} \in \mathbb{R}$ the PWE would be

$$
\tilde{u}\left(\boldsymbol{K}, z_{o}\right)=A_{p} \delta\left(\boldsymbol{K}-k_{o} \mathbf{1}_{t} \cdot \hat{\boldsymbol{k}}_{p}\right) .
$$

In case of $k_{o} \in \mathbb{C}$ and $\boldsymbol{K} \in \mathbb{R}^{2}$, it is no longer possible to satisfy this condition, as Dirac's delta is never evaluated at its singularity in $k_{o} \hat{\boldsymbol{k}}_{p}$, now outside the real plane spanned by $\boldsymbol{K}$ in the standard Fourier-based PWE. Moreover, precise identification requires sampling having access to the entire plane $z=0$, which then would imply that homogeneous plane waves in a lossy medium would exponentially diverge. Fourier transforms cannot be applied to this kind of functions, but we can still consider the case where only a portion of the $z=0$ plane is sampled, as it happens in practical applications of the PWE, e.g., for measured data.

For a single homogeneous plane wave of amplitude $A_{p}$ propagating along $\hat{\boldsymbol{k}}=\hat{\boldsymbol{x}} \sin \theta_{p}+\hat{\boldsymbol{z}} \cos \theta_{p}$, its PWE would be estimated as in (3), limiting the integral over a square region of side $2 a$, yielding

$$
\begin{aligned}
& \tilde{u}\left(\boldsymbol{K}, z_{o}\right)=A_{p} \frac{2 a \operatorname{sinc}\left(k_{y} a\right)}{k_{o} \sin \theta_{p}-k_{x}}\left[\sin \left(\Delta k_{x}^{\prime} a\right) \cosh \left(k_{o}^{\prime \prime} a \sin \theta_{p}\right)+\right. \\
& \left.\mathrm{j} \cos \left(\Delta k_{x}^{\prime} a\right) \sinh \left(k_{o}^{\prime \prime} a \sin \theta_{p}\right)\right]
\end{aligned}
$$

where $\Delta k_{x}^{\prime}=k_{o}^{\prime} \sin \theta_{p}-k_{x}$ and $k_{o}=k_{o}^{\prime}+\mathrm{j} k_{o}^{\prime \prime}$. For a lossless medium the above formula would have a vanishing imaginary part, leaving a sinc function centered at $\boldsymbol{K}_{p}=\left(k_{o} \sin \theta_{p}, 0\right)$, converging to a Dirac's distribution as $a \rightarrow \infty$, i.e., as in (13). Computing the square modulus of (14)

$$
\left|\tilde{u}\left(\boldsymbol{K}, z_{o}\right)\right|^{2}=2 a^{2}\left|A_{p}\right|^{2} \operatorname{sinc}^{2}\left(k_{y} a\right) \frac{\cosh \left(2 k_{o}^{\prime \prime} a \sin \theta_{p}\right)-\cos \left(2 \Delta k_{x}^{\prime} a\right)}{\left(\Delta k_{x}^{\prime}\right)^{2}+\left(k_{o}^{\prime \prime} \sin \theta_{p}\right)^{2}}
$$

it appears that the peak amplitude is still found at $\boldsymbol{K}_{p}$, where $\Delta k_{x}^{\prime}=0$, even as $k_{o}^{\prime \prime} \neq 0$, when $\operatorname{Re} \tilde{u}\left(\boldsymbol{K}_{p}, z_{o}\right)=0$. The fact that it is the imaginary part of (14) that provides the identification of the plane wave is an issue, as it does not behave as an approximation of a Dirac's distribution, as it was the case for the lossless case. Moreover, the value taken by (15)

$$
\tilde{u}\left(\boldsymbol{K}_{p}, z_{o}\right)=\mathrm{j} 2 a A_{p} \frac{\sinh \left(k_{o}^{\prime \prime} a \sin \theta_{p}\right)}{k_{o}^{\prime \prime} a \sin \theta_{p}}
$$

is in quadrature with the actual amplitude $A_{p}$ and depends on $k_{o}^{\prime \prime}$, quickly diverging as $k_{o}^{\prime \prime} a \sin \theta_{p}$ increases, whereas in lossless conditions increasing $a$ has no impact on estimating $A_{p}$. 
Therefore, only for $k_{o}^{\prime \prime} \rightarrow 0$ (14) yields an exact estimate of the plane wave amplitude.

This example shows a fundamental limitation of the standard definition of the PWE, since for a single homogeneous plane wave it cannot identify its parameters correctly. As a result, its PWE cannot be interpreted as an estimate of the physical phenomena underlying the sampled field distribution, even though inverse-transforming (14) the original field distribution is correctly retrieved.

\section{Generalized plane-wave expansions and propagators}

The observations presented in the previous section can be ascribed to the arbitrary choice of only using $\boldsymbol{K} \in \mathbb{R}^{2}$. As we will discuss at the end of this section, this choice is correct and actually necessary in the case $k_{o} \in \mathbb{R}$, but it is not justified either mathematically or physically for the more general case of lossy media, where $k_{o} \in \mathbb{C}$.

Choosing $\boldsymbol{K} \in \mathbb{C}^{2}$ means switching from Fourier functions to those used in Laplace transform, i.e., for a generic complex $\boldsymbol{K}=\boldsymbol{K}^{\prime}+\mathrm{j} \boldsymbol{K}^{\prime \prime}$

$$
\mathrm{e}^{-\mathrm{j} \boldsymbol{K} \cdot \boldsymbol{R}}=\mathrm{e}^{\boldsymbol{K}^{\prime \prime} \cdot \boldsymbol{R}} \mathrm{e}^{-\mathrm{j} \boldsymbol{K}^{\prime} \cdot \boldsymbol{R}} .
$$

Adopting the standard definition of Laplace transform, the natural candidate for the Laplace variable in the spectral domain would be $\mathrm{j} \boldsymbol{K}=\mathrm{j} \boldsymbol{K}^{\prime}-\boldsymbol{K}^{\prime \prime}$, with a generalized PWE defined as

$$
\tilde{u}(\boldsymbol{K}, z)=\int_{-\infty}^{\infty} \int_{-\infty} \mathrm{d}\left[\tilde{u}(\boldsymbol{R}, z) \mathrm{e}^{-\boldsymbol{K}^{\prime \prime} \cdot \boldsymbol{R}}\right] \exp \left(\mathrm{j} \boldsymbol{K}^{\prime} \cdot \boldsymbol{R}\right) .
$$

Laplace transform is better known for applications to functions of time, where it is only applied to positive time values, since any real system can be assumed to be causal. There is no such constraint in the case of spatial field distributions analyzed in harmonic steady-state conditions, where they cover in a general way the entire plane. Therefore, for the case at hand here, it will be necessary to consider the two-sided Laplace transform. The consequences of this choice are discussed later in this section, when looking for regions of convergence.

The main advantage with respect to Fourier functions is their ability to introduce an exponential weighting, controlled by $\boldsymbol{K}^{\prime \prime}$, which allows dealing with exponentially diverging functions, such as in the case of a single homogeneous plane wave. In practice, the exponential $\exp \left(\boldsymbol{K}^{\prime \prime} \cdot \boldsymbol{R}\right)$ operates as a normalization of the spatial distribution, capable of compensating exponential divergence in the spatial data, as is the case for plane waves in homogeneous lossy media. Moreover, switching to Laplace transform also allows sampling the PWE at $\boldsymbol{K} \in \mathbb{C}^{2}$, as required by (12), in order to accurately predict far-field radiation.

At first glance Laplace transform could be regarded as burdensome with respect to Fourier transform, as the imaginary parts of $k_{x}=k_{x}^{\prime}+\mathrm{j} k_{x}^{\prime \prime}$ and $k_{y}=k_{y}^{\prime}+\mathrm{j} k_{y}^{\prime \prime}$ now also need to be explored, thus passing from a two-dimensional to a practically four-dimensional function. In fact, there is no need to explore all possible values of $\boldsymbol{K}^{\prime \prime}$, since passing back to the spatial domain by performing an inverse Laplace transform requires choosing only a single value of $\boldsymbol{K}^{\prime \prime}$, or Laplace cut, as required by Bromwich integral [14],

$$
u(\boldsymbol{R}, z)=(2 \pi \mathrm{j})^{-2} \int_{k_{x}^{\prime \prime}-\mathrm{j} \infty}^{k_{x}^{\prime \prime}+\mathrm{j} \infty} \mathrm{d}\left(\mathrm{j} k_{x}^{\prime}\right) \int_{k_{y}^{\prime \prime}-\mathrm{j} \infty}^{k_{y}^{\prime \prime}+\mathrm{j} \infty} \mathrm{d}\left(\mathrm{j} k_{y}^{\prime}\right) \tilde{u}(\boldsymbol{K}, z) \mathrm{e}^{-\mathrm{j} \boldsymbol{K} \cdot \boldsymbol{R}},
$$

which can be recast as

$$
u(\boldsymbol{R}, z)=\frac{\mathrm{e}^{\boldsymbol{K}^{\prime \prime} \cdot \boldsymbol{R}}}{4 \pi^{2}} \iint_{-\infty}^{\infty} \mathrm{d} \boldsymbol{K}^{\prime} \tilde{u}\left(\boldsymbol{K}^{\prime}+\mathrm{j} \boldsymbol{K}^{\prime \prime}, z\right) \exp \left(-\mathrm{j} \boldsymbol{K}^{\prime} \cdot \boldsymbol{R}\right) .
$$

The Laplace pair as defined by (18) and (20) shows that passing to Laplace transform still involves computing Fourier transforms, but now applied to a spatial distribution undergoing a normalization as it is weighted by a real-argument exponential decaying along the direction $\boldsymbol{K}^{\prime \prime}$. The chosen value of $\boldsymbol{K}^{\prime \prime}$ must belong to the region of convergence (ROC) of the Laplace transform. The ROC can be identified by looking for the set of $\boldsymbol{K}^{\prime \prime}$ such that

$$
\lim _{R \rightarrow \infty}\left|\mathrm{e}^{\boldsymbol{K}^{\prime \prime} \cdot \boldsymbol{R}} u(\boldsymbol{R}, z)\right|<M \quad \forall \hat{\boldsymbol{R}}
$$

where $M$ is a finite real number. The above condition, applied to all directions $\hat{\boldsymbol{R}}$ over the $x y$ plane, translates the requirement for a bounded spatial distribution after weighting $u(\boldsymbol{R}, z)$ by an exponential function. In lossy media, the far-field amplitude of fields generated by finite-size sources is dominated by exponential functions of the imaginary part of $k_{o}[15,16]$. Hence (21) holds as long as

$$
\left(k_{x}^{\prime \prime}\right)^{2}+\left(k_{y}^{\prime \prime}\right)^{2}=\left\|\boldsymbol{K}^{\prime \prime}\right\|^{2}<\left(k_{o}^{\prime \prime}\right)^{2} .
$$

In case of losses, different choices of $\boldsymbol{K}^{\prime \prime}$ yield different definitions of the PWE, with their own propagator, still defined as in (6), with (8) becoming

$$
\gamma^{2}=k_{o}^{2}-\boldsymbol{K}^{\prime} \cdot \boldsymbol{K}^{\prime}-j \boldsymbol{K}^{\prime \prime} \cdot \boldsymbol{K}^{\prime \prime}+2 \mathrm{j} \boldsymbol{K}^{\prime} \cdot \boldsymbol{K}^{\prime \prime},
$$

where $\boldsymbol{K}^{\prime} \cdot \boldsymbol{K}^{\prime \prime} \neq 0$ as opposed to the usual case of physical settings of waves propagating at the interface between lossless and lossy media.

According to Bromwich theorem, any choice of $\boldsymbol{K}^{\prime \prime}$ satisfying (22) will yield identical results when coming back to the spatial domain, after propagation. Hence, there is not a unique definition for the PWE, but rather an infinite number, all of them equivalent when it comes to propagating field distributions from a plane $z_{o}$ to a plane $z$. The two main reasons for choosing $\boldsymbol{K}^{\prime \prime} \neq 0$ are either to compute the far-field radiation associated to the field scanned at $z_{o}$, as required by (11) or in order to identify the exact complex amplitude of a plane wave.

This last case was shown in the previous section to be an issue, with an inaccurate estimate PWE given by (14). This problem can be solved by first computing the PWE for $\boldsymbol{K}^{\prime \prime}=0$, which was shown in Sec. 2.2 to attain its peak intensity at $\boldsymbol{K}_{p}^{\prime}=$ $\left(k_{o}^{\prime} \sin \theta_{p}, 0\right)$. The generalized PWE with Laplace transform for $\boldsymbol{K}^{\prime}=\boldsymbol{K}_{p}^{\prime}$ reduces to

$$
\tilde{u}\left(\boldsymbol{K}_{p}^{\prime}+\mathrm{j} \boldsymbol{K}^{\prime \prime}, z_{o}\right)=A_{p} \iint_{-\infty}^{\infty} \mathrm{d} \boldsymbol{R} \mathrm{e}^{\left(\boldsymbol{K}^{\prime \prime}-k_{o}^{\prime \prime} \sin \theta_{p} \hat{\boldsymbol{x}}\right) \cdot \boldsymbol{R}},
$$



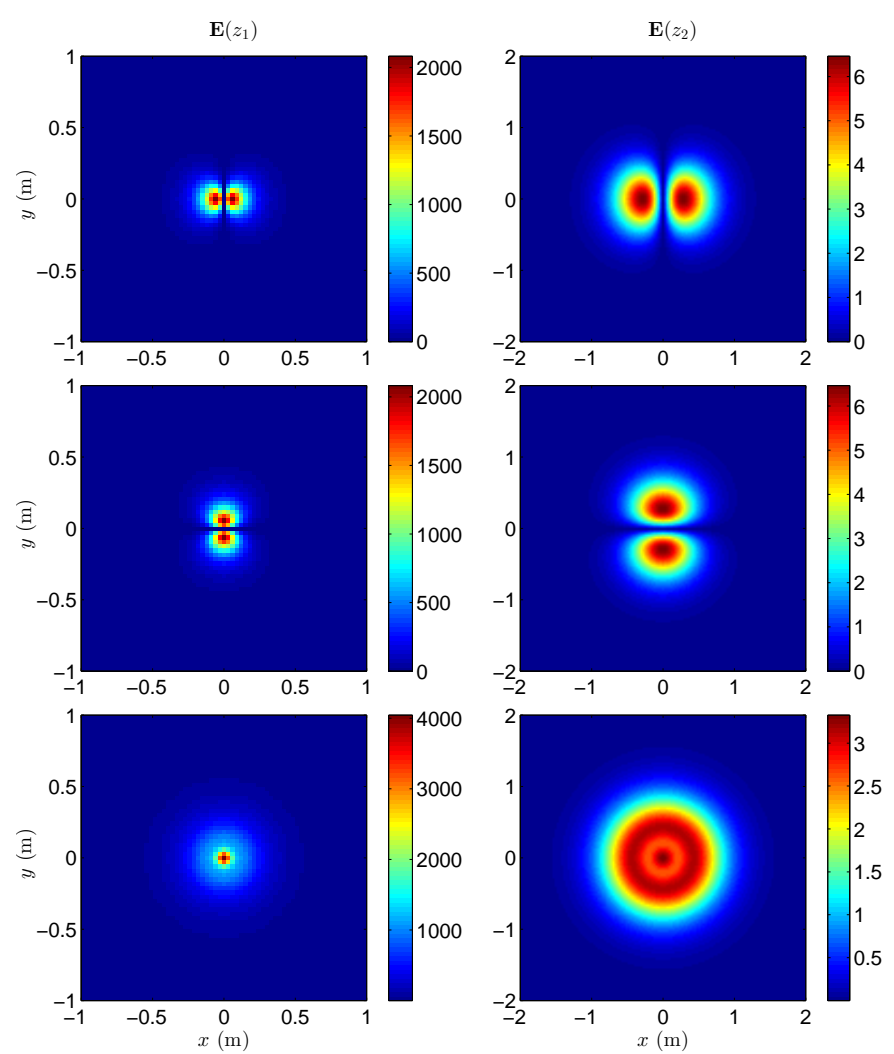

Figure 1: Modulus of field distributions at $z_{1}$ (left column), $z_{2}$ (right column) for an Hertzian dipole normal to the scan plane ( $\hat{z}$-oriented). The three rows represent the Cartesian components of the electric field, $x, y$ and $z$.

which converges only for $\boldsymbol{K}^{\prime \prime}=k_{o}^{\prime \prime} \sin \theta_{p} \hat{\boldsymbol{x}}$, a value that can be predicted since $\theta_{p}$ can be estimated from $\boldsymbol{K}_{p}^{\prime}$ for homogeneous plane waves. Finally, $\tilde{u}\left(\boldsymbol{K}_{p}^{\prime}+\mathrm{j} \boldsymbol{K}_{p}^{\prime \prime}, z_{o}\right)=A_{p} \delta\left(\boldsymbol{K}^{\prime}-\boldsymbol{K}_{p}^{\prime}\right)$, where the amplitude of the plane wave is now clearly identified. This property underlies a recent application of Laplace transform for parameter identification of plane waves in acoustic fields [17].

In the case of lossless media, $u\left(\boldsymbol{R}, z_{o}\right)$ decays as $1 / r$, so any real-argument exponential function would make Laplace integral diverging. In this case therefore the PWE is uniquely defined for $\boldsymbol{K}^{\prime \prime}=0$.

As seen above, Bromwich integral implies that all the choices of $\boldsymbol{K}^{\prime \prime}$ in the ROC (22) are equivalent. Therefore, the apparent inconsistencies summarized in Sec. 2.2 are mainly due to interpreting the individual components of the standard PWE as physical quantities, rather than purely mathematical ones. Physical meaning is more clearly associated to far-field radiation, where the concept of locally-plane waves is unambiguous. In this case, (12) does require to chose $\boldsymbol{K}^{\prime \prime} \neq 0$, where the quantities yielded by the PWE correspond to meaningful amplitudes of plane waves. But as long as field propagation from one plane to another is at stake, any choice of $\boldsymbol{K}^{\prime \prime}$ that satisfies (22) is correct, including $\boldsymbol{K}^{\prime \prime}=0$ for the standard definition, as shown in Sec. 4.
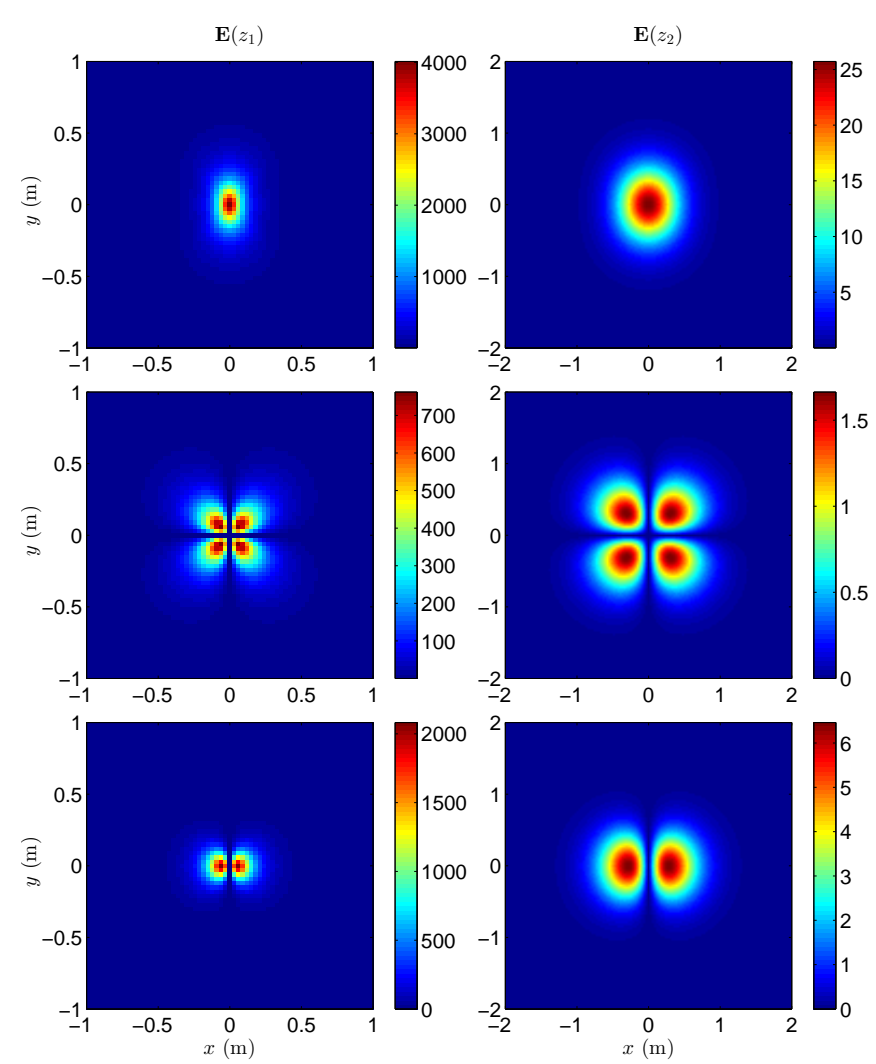

Figure 2: Modulus of field distributions at $z_{1}$ (left column), $z_{2}$ (right column) for an Hertzian dipole tangent to the plane ( $\hat{\boldsymbol{x}}$-oriented). The three rows stand for the Cartesian components of the electric field, $x, y$ and $z$.

\section{The case of an Hertzian dipole}

A Hertzian dipole surrounded by a homogeneous isotropic medium is considered in this section in order to study the behavior of the generalized PWE discussed above. The rationale for this choice is the availability of closed-form formulas for the radiation of Hertzian dipoles, valid even at close range [18]

$$
\begin{aligned}
& E_{r}(\boldsymbol{r})=\frac{p \cos \theta}{2 \pi \mathrm{j} \omega \epsilon_{o} \epsilon_{c} r^{3}}\left(1+\mathrm{j} k_{o} r\right) \mathrm{e}^{-j k_{o} r} \\
& E_{\theta}(\boldsymbol{r})=\frac{p \sin \theta}{4 \pi \mathrm{j} \omega \epsilon_{o} \epsilon_{c} r^{3}}\left[1+\mathrm{j} k_{o} r+\left(\mathrm{j} k_{o} r\right)^{2}\right] \mathrm{e}^{-j k_{o} r} \\
& E_{\phi}(\boldsymbol{r})=0
\end{aligned}
$$

where $p$ is the electric dipole moment of the source. A Cartesian representation will be adopted in the following.

The medium chosen for the analysis has a complex relative dielectric constant $\epsilon_{c}=1-\mathrm{j} 0.5$; the working frequency is set at $1 \mathrm{GHz}$, hence $k_{o}=21.57-\mathrm{j} 5.093 \mathrm{~m}^{-1}$. The electric field radiated by the dipole is sampled at a plane $z_{1}=0.1 \mathrm{~m}$ away from it, and its PWE is employed in order to compute the field radiated at $z_{2}=0.6 \mathrm{~m}$, slightly more than 1.5 wavelengths away from the first plane.

Two orientations of the dipole with respect to the scan plane are considered, namely along $\hat{z}$ (vertical dipole) and $\hat{\boldsymbol{x}}$ (horizontal dipole). The spatial distribution of the three Cartesian 


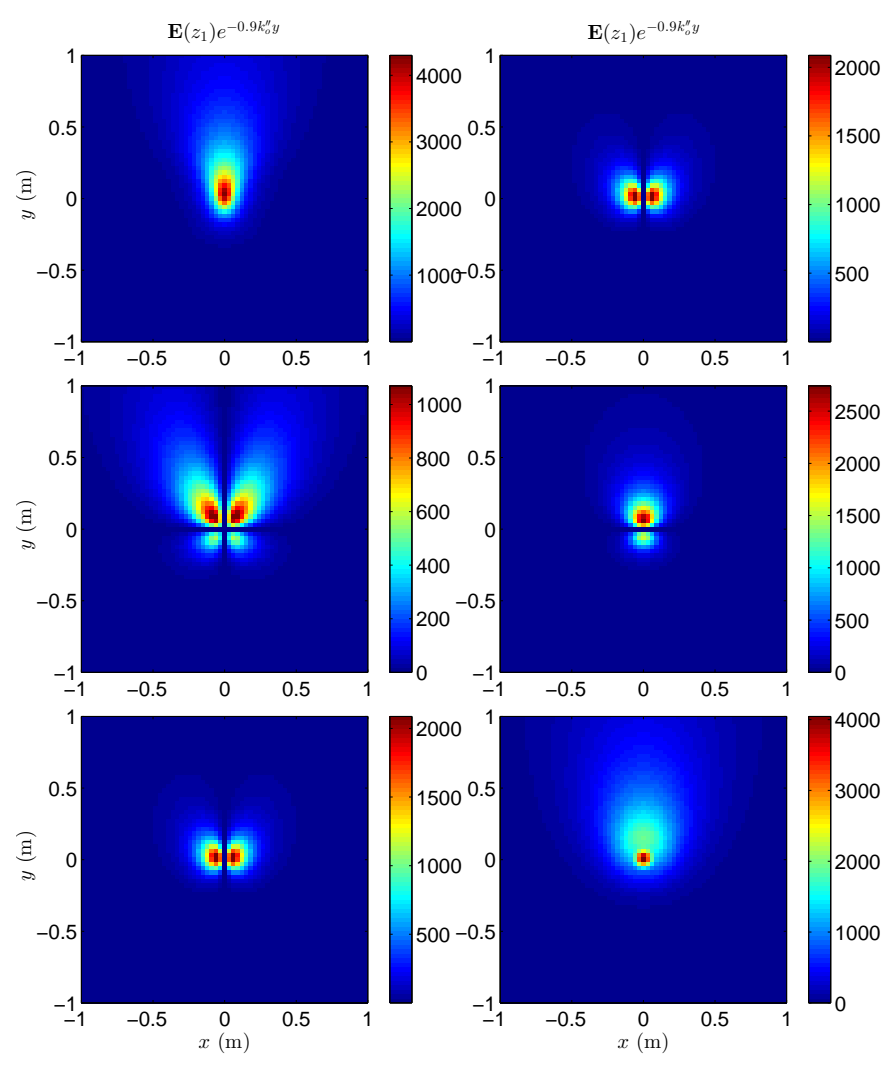

Figure 3: Modulus of field distributions at $z_{1}$ after applying Laplace exponential, for $k_{y}^{\prime \prime} / k_{o}=0.9$. These distributions are those that will be Fourier transformed for generalized PWE. Hertzian dipole tangent to the scan plane $(\hat{\boldsymbol{x}}$ oriented) in the left column, normal to the plane ( $\hat{z}$-oriented) on the right. The three rows stand for the Cartesian components of the electric field, $x, y$ and $z$.

components of $\boldsymbol{E}(\boldsymbol{R}, z)$ are shown in Figs. 1 and 2, for the two planes $z=z_{1}$ and $z=z_{2}$.

Four generalized PWE are considered in the following, by choosing $k_{y}^{\prime \prime} / k_{o}^{\prime \prime}=\{0,0.3,0.6,0.9\}$ and $k_{x}^{\prime \prime}=0$. These choices of the Laplace-domain cut correspond to applying the Fourier transform to the spatial distributions shown in Fig. 3. The corresponding spectral propagators are shown in Fig. 4.

The spectra computed for these four choices of $k_{y}^{\prime \prime}$, shown in Figs. 5 and 6, present strong modifications only for certain Cartesian components. These modifications can be interpreted by recalling that for a homogeneous plane wave with $k_{y}^{\prime \prime}=k_{o}^{\prime \prime}$ it must necessarily present $k_{y}^{\prime}=k_{o}^{\prime}$. As $k_{y}^{\prime \prime} \rightarrow k_{o}^{\prime \prime}$ in Figs. 5 and 6 those components that are expected to subsist in the far-field region of the sources present increasingly strong and resolved peaks around $\boldsymbol{K}=k_{o}^{\prime} \hat{\boldsymbol{y}}$, consistently with the requirement of (12), as proven in [7]. These peaks occur for the $y$ and $z$ components in case of an $z$-oriented dipole, and for the $x$ component for the $x$-oriented one.

A formal explanation can be provided, by computing the generalized PWE of a dipole. Rather than doing this from spatial distributions given in (25), it is easier to proceed from the reciprocal-space (or $\boldsymbol{k}$-space) representation of the electric-
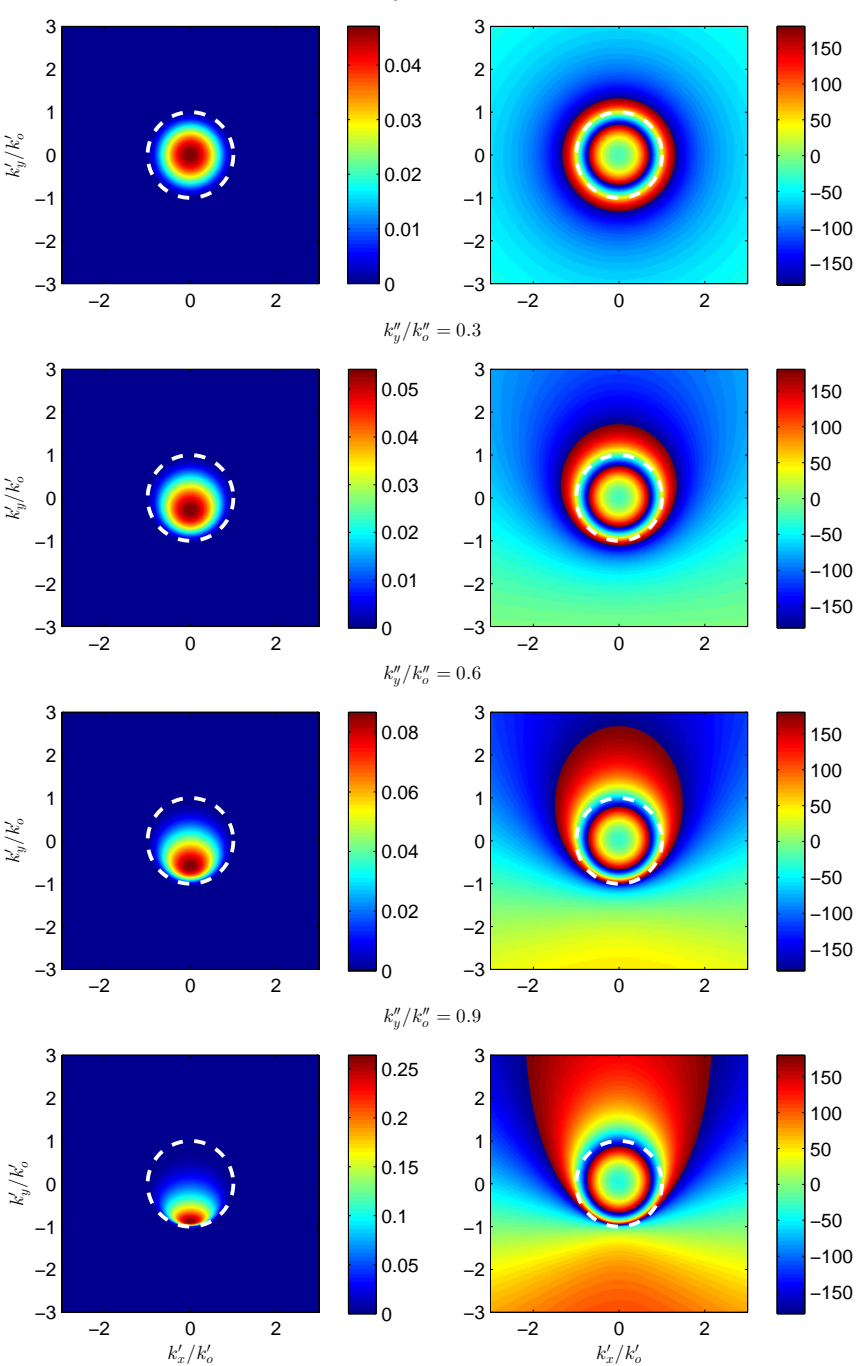

Figure 4: Comparison of generalized propagators computed for $z_{2}-z_{1}$ for four choices of $k_{y}^{\prime \prime}$. Modulus (left column), phase in radians (right column).

electric Green function [6]

$$
\tilde{\boldsymbol{G}}(\boldsymbol{k})=\frac{\boldsymbol{1}-k_{o}^{2} \boldsymbol{k} \boldsymbol{k}}{k^{2}-k_{o}^{2}}=\frac{\tilde{\boldsymbol{D}}(\boldsymbol{k})}{k^{2}-k_{o}^{2}}
$$

with 1 the identity dyad. Given an electric-current density distribution $\boldsymbol{J}(\boldsymbol{r})$ whose $\boldsymbol{k}$-space representation is $\tilde{\boldsymbol{J}}(\boldsymbol{k})$

$$
\tilde{\boldsymbol{E}}(\boldsymbol{k})=-\mathrm{j} \omega \mu \tilde{\boldsymbol{G}}(\boldsymbol{k}) \cdot \tilde{\boldsymbol{J}}(\boldsymbol{k}) .
$$

In case of a Hertzian dipole oriented along $\hat{\boldsymbol{p}}, \boldsymbol{J}(\boldsymbol{r})=\delta(\boldsymbol{r}) \hat{\boldsymbol{p}}$, hence $\tilde{\boldsymbol{J}}(\boldsymbol{k})=\hat{\boldsymbol{p}}$.

The PWE can then be computed as

$$
\tilde{\boldsymbol{E}}(\boldsymbol{K}, z)=\frac{1}{2 \pi} \int \mathrm{d} k_{z} \tilde{\boldsymbol{E}}(\boldsymbol{k}) \mathrm{e}^{-\mathrm{j} k_{z} z},
$$

i.e., inverse transforming only along the $k_{z}$ dimension. Carrying out the integration for (27) using Cauchy theorem and choosing 
an integration path in order to only include forward propagating waves yields

$$
\tilde{\boldsymbol{E}}(\boldsymbol{K}, 0)=\frac{\mathrm{j}}{2 \gamma} \tilde{\boldsymbol{D}}(\boldsymbol{K}+\gamma \hat{\boldsymbol{z}}) \cdot \hat{\boldsymbol{p}}
$$

where $\gamma$ was defined in (23).

For the case of a vertical dipole oriented along $\hat{z}$ (29) simplifies to

$$
\tilde{\boldsymbol{E}}(\boldsymbol{K}, 0)=\frac{\mathrm{j}}{2 \gamma k_{o}^{2}}\left(\begin{array}{c}
k_{x} \gamma \\
k_{y} \gamma \\
1-\gamma^{2}
\end{array}\right),
$$

i.e., presenting a singular $z$ component for $\gamma=0$, i.e., for all $\boldsymbol{K}$ such that $\boldsymbol{K} \cdot \boldsymbol{K}=k_{o}^{2}$. This condition is satisfied when $\left\|\boldsymbol{K}^{\prime}\right\|=$ $k_{o}^{\prime}$ and $\left\|\boldsymbol{K}^{\prime \prime}\right\|=k_{o}^{\prime \prime}$, i.e., for grazing propagation along the scan plane, and is consistent with the peaks observed earlier in this section in Fig. 5. The consequence is that these peaks are in fact singularities that, once passing back to space through spectral integration, correspond to plane wave contributions.

In the same way, for an $\hat{x}$-oriented dipole,

$$
\tilde{\boldsymbol{E}}(\boldsymbol{K}, 0)=\frac{\mathrm{j}}{2 \gamma k_{o}^{2}}\left(\begin{array}{c}
k_{o}^{2}-k_{x} \\
-k_{x} k_{y} \\
k_{x} \gamma
\end{array}\right),
$$

which is now singular for the $x$ and $y$ components of the PWE, again for grazing directions along the scan plane, consistent with the peaks observed in Fig. 6.

More simply stated, the choice of a Laplace cut different from the one used in the Fourier transform naturally leads to a dominant contribution in the generalized PWE, which happens to coincide with a homogeneous plane wave, as expected for a source radiating in a homogeneous medium. The standard definition of PWE would not allow observing these singularities, as they occur outside the Fourier cut $\boldsymbol{K}^{\prime \prime}=0$.

Once the four PWE are propagated at $z_{2}$ and inverse transformed according to (20), the results are indistinguishable from the references shown in Figs. 5 and 6, as expected from Bromwich theorem, with errors below $0.01 \%$ of the respective peak amplitudes due to finite numerical resolution.

\section{Conclusions}

This paper has argued about apparent inconsistencies observed when extending the standard Fourier-based definition of the PWE to lossy media. The inaccurate identification of homogeneous plane waves was pointed out as an intrinsical limitation. Switching to a more general Laplace-based definition was shown to reintroduce homogeneous plane waves, which are needed in order to describe the asymptotic evolution of far-field radiation.
The fact that the generalized definition allows multiple choices for $\boldsymbol{K}^{\prime \prime}$ has direct implications on the interpretation of PWE. Since any choice of Laplace cut must yield identical results, it is no longer possible to define a single interpretation of the PWE, as each one is based on a different set of plane waves with complementary properties. While the resulting field distributions are identical by virtue of Bromwich theorem, the expansions no longer represent a collection of functions that can be interpreted on an individual basis. The discussions presented in this paper stress the fact that the PWE should be regarded only as a mathematical representation, restraining from interpreting its individual components as physical entities.

\section{References}

[1] P. Clemmow, The plane wave spectrum representation of electromagnetic fields, Pergamon Press (Oxford and New York), 1966.

[2] D. M. Kerns, Plane-wave scattering-matrix theory of antennas and antenna-antenna interactions, NASA STI/Recon Technical Report N 82 (1981) 15358.

[3] J. J. Stamnes, Waves in focal regions: propagation, diffraction and focusing of light, sound and water waves, CRC Press, 1986.

[4] C. Scott, The spectral domain method in electromagnetics, Norwood, MA, Artech House, 1989, 149 p. 1.

[5] M. Nieto-Vesperinas, Scattering and diffraction in physical optics, Wiley New York, 1991.

[6] L. B. Felsen, N. Marcuvitz, Radiation and scattering of waves, Vol. 31, John Wiley \& Sons, 1994.

[7] T. Hansen, A. D. Yaghjian, Plane-wave theory of time-domain fields, IEEE Press, 1999.

[8] A. J. Devaney, Mathematical foundations of imaging, tomography and wavefield inversion, Cambridge University Press, 2012.

[9] G. C. Sherman, A. Devaney, L. Mandel, Plane-wave expansions of the optical field, Optics Communications 6 (2) (1972) 115-118.

[10] G. C. Sherman, J. J. Stamnes, A. Devaney, É. Lalor, Contribution of the inhomogeneous waves in angular-spectrum representations, Optics Communications 8 (4) (1973) 271-274.

[11] E. Wolf, J. T. Foley, Do evanescent waves contribute to the far field?, Optics letters 23 (1) (1998) 16-18.

[12] M. Xiao, Evanescent fields do contribute to the far field, journal of modern optics 46 (4) (1999) 729-733.

[13] D. K. Cheng, et al., Field and wave electromagnetics, Pearson Education India, 1989.

[14] W. R. Le Page, Complex Variables and the Laplace Transform for Engineers, Dover Publication Inc., 1980.

[15] F. Atkinson, On Sommerfeld's "radiation condition.", The London, Edinburgh, and Dublin Philosophical Magazine and Journal of Science 40 (305) (1949) 645-651.

[16] S. H. Schot, Eighty years of Sommerfeld's radiation condition, Historia mathematica 19 (4) (1992) 385-401.

[17] A. Geslain, S. Raetz, M. Hiraiwa, M. Abi Ghanem, S. Wallen, A. Khanolkar, N. Boechler, J. Laurent, C. Prada, A. Duclos, et al., Spatial laplace transform for complex wavenumber recovery and its application to the analysis of attenuation in acoustic systems, Journal of Applied Physics 120 (13) (2016) 135107.

[18] J. R. Wait, Electromagnetic wave theory, Harper \& Row, 1985. 

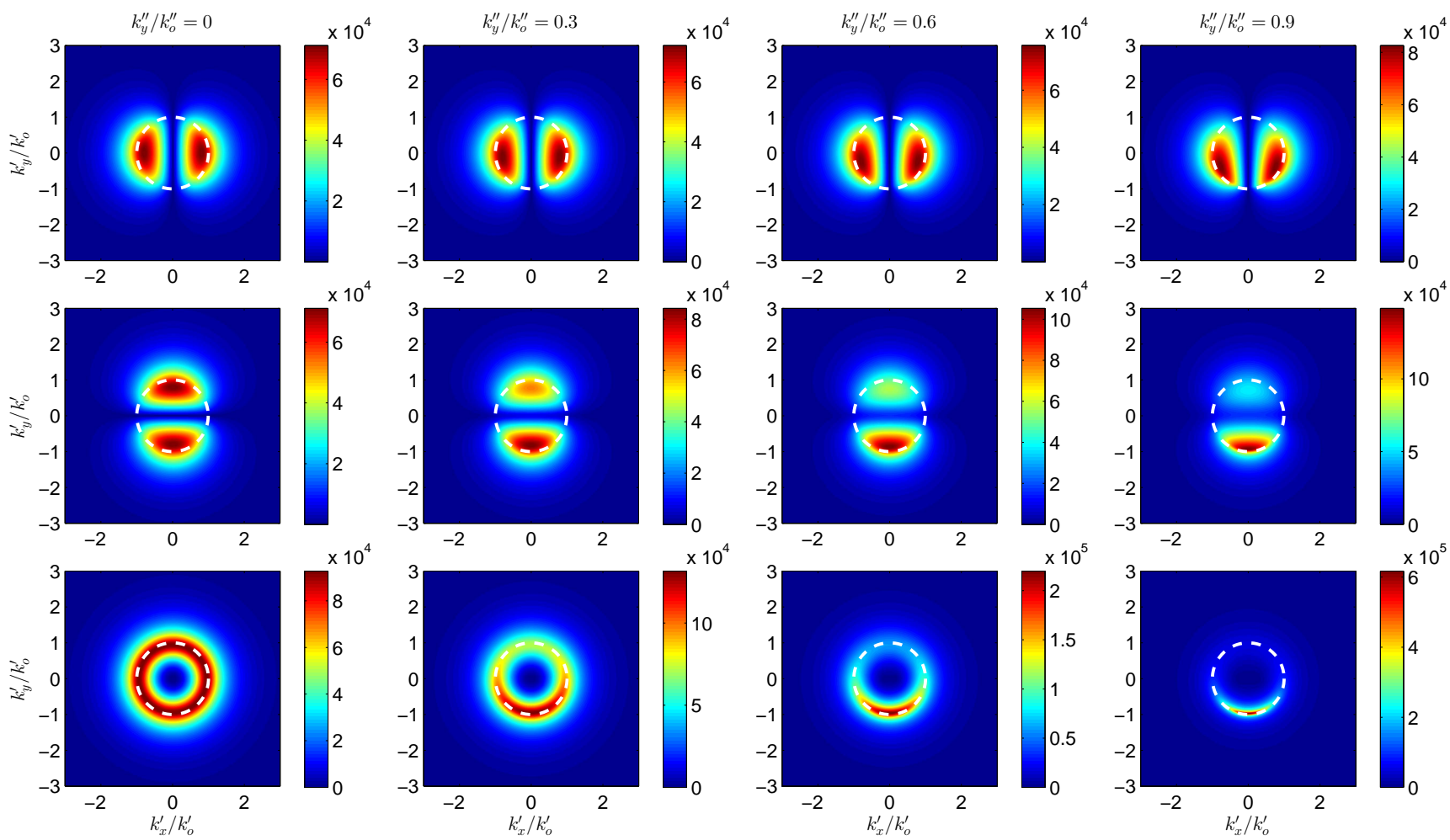

Figure 5: Comparison of generalized PWE computed at $z_{1}$ for four choices of $k_{y}^{\prime \prime}$, for a $z$-oriented Hertzian dipole. From top to bottom row, the three Cartesian components, $x, y$ and $z$.
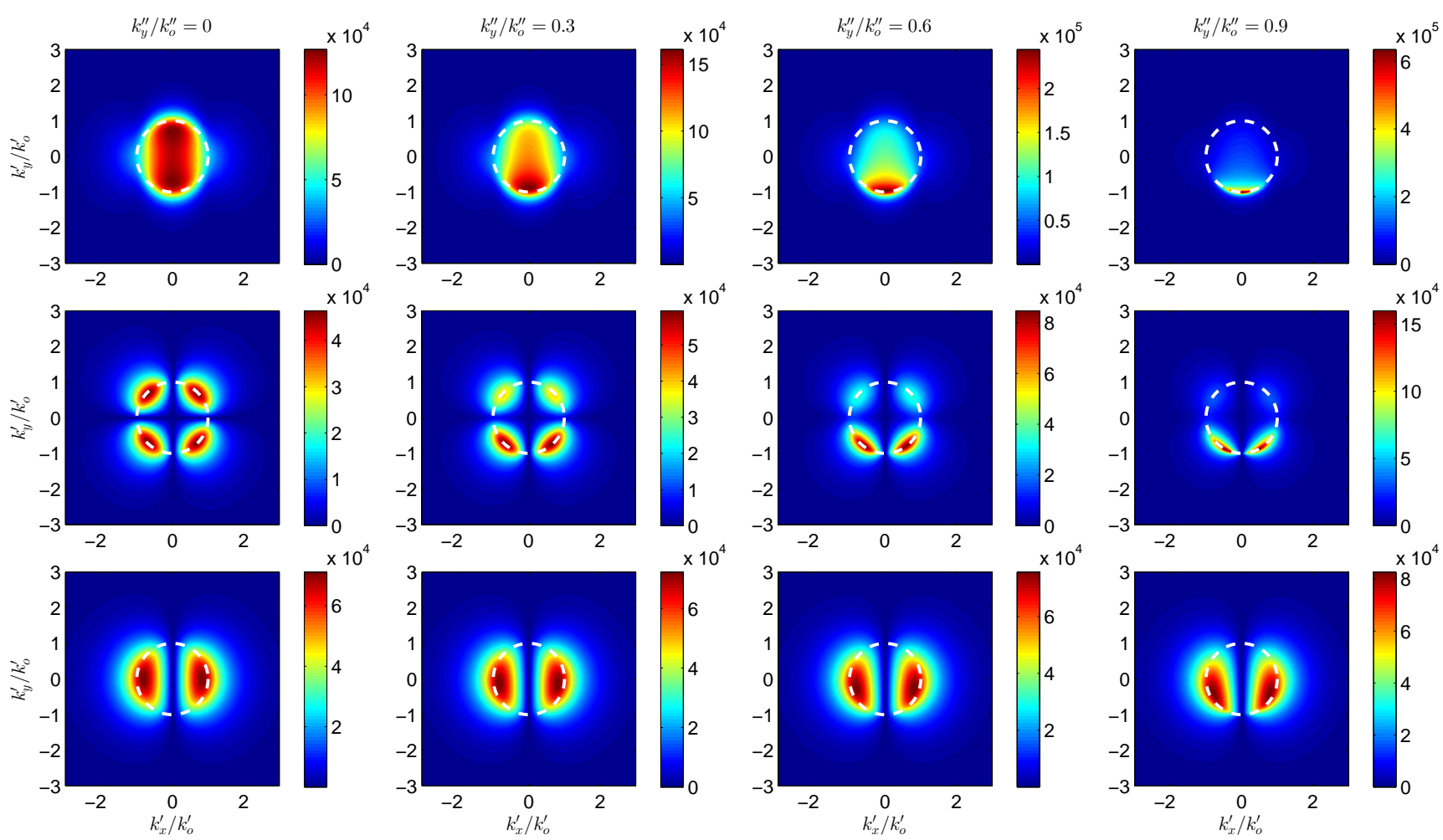

Figure 6: Comparison of generalized PWE computed at $z_{1}$ for four choices of $k_{y}^{\prime \prime}$, for a $y$-oriented Hertzian dipole. From top to bottom row, the three Cartesian components, $x, y$ and $z$. 